\title{
OPEN Altered amygdala-based functional connectivity in individuals with attenuated psychosis syndrome and first-episode schizophrenia
}

\author{
Woo-Sung Kim ${ }^{1}$, Guangfan Shen ${ }^{1}$, Congcong Liu ${ }^{1}$, Nam-In Kang ${ }^{4}$, Keon-Hak Lee ${ }^{4}$, \\ Jing Sui ${ }^{5,6}$ \& Young-Chul Chung ${ }^{1,2,3 凶}$
}

Altered resting-state functional connectivity (FC) of the amygdala (AMY) has been demonstrated to be implicated in schizophrenia (SZ) and attenuated psychosis syndrome (APS). Specifically, no prior work has investigated FC in individuals with APS using subregions of the AMY as seed regions of interest. The present study examined AMY subregion-based FC in individuals with APS and firstepisode schizophrenia (FES) and healthy controls (HCs). The resting state FC maps of the three AMY subregions were computed and compared across the three groups. Correlation analysis was also performed to examine the relationship between the Z-values of regions showing significant group differences and symptom rating scores. Individuals with APS showed hyperconnectivity between the right centromedial AMY (CMA) and left frontal pole cortex (FPC) and between the laterobasal AMY and brain stem and right inferior lateral occipital cortex compared to HCs. Patients with FES showed hyperconnectivity between the right superficial AMY and left occipital pole cortex and between the left CMA and left thalamus compared to the APS and HCs respectively. A negative relationship was observed between the connectivity strength of the CMA with the FPC and negative-others score of the Brief Core Schema Scales in the APS group. We observed different altered FC with subregions of the AMY in individuals with APS and FES compared to HCs. These results shed light on the pathogenetic mechanisms underpinning the development of APS and SZ.

Schizophrenia (SZ) is a severe neuropsychiatric disorder. A leading hypothesis is that SZ is a brain disconnection syndrome involving abnormal interactions among widespread brain networks ${ }^{1,2}$. Widespread network disconnectivity in SZ has been reported using both seed-based analysis and independent component analysis (ICA) with rs-fMRI data (for a review, see Ref. ${ }^{3}$ ). More specifically, most seed-based studies have found decreased connectivity $^{4-7}$, whereas ICA studies have reported both increases ${ }^{8,9}$ and decreases ${ }^{10,11}$. The medial frontal cortex (mPFC) has often been shown by both approaches to be involved in aberrant connectivity in $\mathrm{SZ}^{3}$. The main findings of rs-fMRI studies in first-episode schizophrenia (FES) are similar. Robust regional brain changes were found in the prefrontal and temporal lobes, including decreased functional connectivity (FC) in the mPFC ${ }^{12,13}$, dorsal lateral prefrontal cortex (DLPFC) ${ }^{14}$, orbital frontal cortex ${ }^{13}$, and ventrolateral prefrontal cortex ${ }^{13,14}$ and increased or decreased FC in the temporal lobe, with prominence in the left superior temporal gyrus (STG) ${ }^{15,16}$ (for a review, see Ref. $^{17}$ ).

Individuals with attenuated psychosis syndrome (APS), the most common type of clinical high risk (CHR) state for psychosis, provide an opportunity to explore the earliest pathophysiology of SZ given the consistent

\footnotetext{
${ }^{1}$ Department of Psychiatry, Medical School, Jeonbuk National University, Geonjiro 20, Jeonju, Korea. ${ }^{2}$ Department of Psychiatry, Jeonbuk National University Hospital, Jeonju, Korea. ${ }^{3}$ Research Institute of Clinical Medicine of Jeonbuk National University-Biomedical Research Institute of Jeonbuk National University Hospital, Jeonju, Korea. ${ }^{4}$ Department of Psychiatry, Maeumsarang Hospital, Wanju, Jeollabuk-do, Korea. ${ }^{5}$ Brainnetome Center and National Laboratory of Pattern Recognition, Institute of Automation, Chinese Academy of Sciences, Beijing 100190, China. ${ }^{6}$ University of Chinese Academy of Sciences, CAS Center for Excellence in Brain Science and Intelligence Technology, Chinese Academy of Sciences, Beijing 100049, China. ${ }^{\bowtie}$ email: chungyc@jbnu.ac.kr
} 
transition risk of $22 \%$ after 1 year and $36 \%$ after 3 years $^{18}$. Studies on the resting-state FC (rsFC) in subjects with APS is ongoing, and evidence is limited. Most previous FC studies have investigated networks seeded from a priori defined regions of interest (ROIs): the amygdala (AMY $)^{19}$, cerebellum ${ }^{20}$, posterior cingulate cortex $(\mathrm{PCC})^{21}$, striatum $(\mathrm{ST})^{22,23}, \mathrm{STG}^{24}$, and thalamus ${ }^{25}$. Overall, the data points to reduced FC in the corticolimbic and corticostriatal circuits and mixed findings pertaining to FC in the corticothalamic circuits.

The AMY is composed of structurally and functionally distinct nuclei that contribute to the processing of emotion through interactions with other subcortical and cortical structures. The laterobasal AMY (LBA) facilitates associative learning processes such as fear conditioning through afferent nerves from cortical and subcortical regions, including the thalamus, hippocampus, and prefrontal cortex ${ }^{26,27}$. The centromedial AMY (CMA) plays an important role in generating behavioral responses through projections to the brainstem, as well as cortical and striatal regions ${ }^{26,28}$, and facilitates attention to salient stimuli ${ }^{29}$. The superficial AMY (SA) lies adjacent to the laterobasal group and includes the cortical nuclei involved in olfactory $y^{30,31}$ and affective processes ${ }^{32}$. Despite the potential importance of understanding AMY-related dysfunction in APS and SZ, relatively few studies have directly examined alterations in AMY connectivity across the illness phases; only five studies on SZ ${ }^{19,33-36}$ and two studies on APS ${ }^{19,37}$ have done so. Of note, only two studies on SZ used subregions of the AMY as a seed $\mathrm{ROI}^{34,36}$. Given the different roles of AMY subregions and the aberrant rsFC of specific AMY subregions in SZ, it is crucial to investigate rsFC in the AMY subregions of individuals with APS. To our knowledge, no prior work has investigated FC in individuals with APS using subregions of the AMY as a seed ROI.

We hypothesized that participants with APS would exhibit altered FC between subregions of the AMY and other brain regions compared to healthy controls (HCs) and patients with SZ. The present study examined AMY subregion-based connectivity in individuals with APS, patients with FES, and HCs. The rsFC maps of the three AMY subregions were computed and compared across the three groups. Given the role of AMY in the formation of self-referential schemas ${ }^{38}$ and the close association between negative schemas and positive symptoms s $^{39}$, exploratory analyses were performed to examine the correlations between the Z-values of regions showing significant group differences and Positive and Negative Syndrome Scale (PANSS; Refs. ${ }^{40,41}$ and Brief Core Schema Scales (BCSS; Ref. ${ }^{42}$ ) scores.

\section{Results}

Demographic and clinical characteristics. The FES group comprised nine patients with SZ and 16 patients with schizophreniform disorder. All individuals with APS were classified as the attenuated psychosis group with subthreshold intensity. There were significant differences in sex, education, and BCSS score among the three groups. Post hoc results showed that education level was significantly lower in the APS and FES groups compared to the HC or FES group. On the BCSS, subscale scores of the APS and FES groups were significantly lower than those of the HC group. On the PANSS, total and subscale scores were all significantly lower in the APS group compared to the FES group (Table 1).

AMY seed-based functional connectivity. In the post hoc pairwise comparisons, the APS group showed significantly increased FC between the right CMA and left frontal pole cortex (FPC) $(t=4.84, p<0.05)$ and between the right LBA and brain stem $(t=5.67, p<0.05)$ as well as the right inferior lateral occipital cortex (LOC) compared to HCs. The APS group also exhibited significantly decreased FC between the right CMA and right FPC $(t=5.32, p<0.05)$. On the other hand, the APS group exhibited decreased FC between the right SA and the left occipital pole cortex (OPC) $(t=5.53, \mathrm{p}<0.05)$ compared to the FES group. The FES group exhibited significantly increased FC between the left CMA and left thalamus $(t=5.98, \mathrm{p}<0.05)$ and decreased FC between the right SA and right cerebellum crus $1(t=4.93, \mathrm{p}<0.01)$ (Table 2 and Fig. 1).

Correlations between altered functional connectivity and clinical variables. In the APS group, the mean Z-value of the right CMA and the left FPC connectivity were negatively correlated with the BCSS negative-others score $(r=-0.58, p=0.007)$ (Fig. 2). However, there were no significant correlations between altered FC and clinical variables in the FES group.

\section{Discussion}

The AMY is a key component of a neural system specialized for rapid and automatic evaluation of stimuli that signal potential threat or danger in the environment. Typically, reduced AMY volume and activation to emotional stimuli have been reported in SZ. Using three subregions of the AMY as seed ROIs, we examined rsFC in individuals with APS, patients with FES, and HCs. Aberrant FC with the CMA was much wider and more prominent in the APS group, whereas disconnectivity with the LBA was only evident in SZ.

Individuals with APS showed increased connectivity between the right CMA and left FPC compared to HCs. An earlier study of individuals at high risk for SZ identified no significantly altered FC relative to HCs using the AMY as a single ROI ${ }^{19}$. However, if three subregions of the AMY had been used as seed ROIs, the results may have been different. The CMA plays a significant role in regulating attentional processing of cues during associative conditioning and generating emotional and associated physiological responses to threat or pain through projections to the brainstem, as well as the cortical and striatal regions ${ }^{26,28}$. Stimulation of the central nucleus of the AMY leads to fast, desynchronized cortical EEG activity, which is associated with increased attention and vigilance ${ }^{29,43}$. Projections from the central nucleus to the ventral tegmental area mediate stress-induced increases in dopamine metabolites in the prefrontal cortex ${ }^{44}$. The FPC occupying the most anterior part of the prefrontal cortex is functionally correlated with the default mode network ${ }^{45}$. Its role is specialized for disengaging cognitive control from the current task and re-distributing cognitive resources to other novel goals present in the environment ${ }^{46}$. At-risk and symptomatic individuals for psychosis show abnormalities in both structure ${ }^{47}$ and 


\begin{tabular}{|c|c|c|c|c|}
\hline Characteristics & APS $(n=22)$ & FES $(n=25)$ & HC $(n=31)$ & $p$-value ${ }^{a}$ \\
\hline Age (years) & $22.41(5.16)$ & $24.48(3.45)$ & $24.35(2.35)$ & 0.102 \\
\hline \multicolumn{5}{|l|}{ Sex } \\
\hline Male (n) & 19 & 9 & 14 & \multirow{2}{*}{$0.001^{b}$} \\
\hline Female (n) & 3 & 16 & 17 & \\
\hline Education (years) & $12.09(1.60)^{* * *,+\dagger \dagger}$ & $13.92(1.93)^{\dagger \dagger}$ & $15.29(1.07)$ & $<0.001$ \\
\hline Duration of illness (months) & $24.64(23.60)^{* \star \star}$ & $6.70(7.19)$ & - & $0.001^{\mathrm{c}}$ \\
\hline \multicolumn{5}{|l|}{ SOPS } \\
\hline Positive symptoms & $9.00(9.60)$ & - & - & - \\
\hline Negative symptoms & $9.00(7.26)$ & - & - & - \\
\hline Disorganization symptoms & $1.73(1.93)$ & - & - & - \\
\hline General symptoms & $5.64(3.08)$ & - & - & - \\
\hline Total score & $25.36(16.72)$ & - & - & - \\
\hline \multicolumn{5}{|l|}{ PANSS } \\
\hline Positive symptoms & $9.95(4.20)^{* * *}$ & $17.37(7.10)$ & - & $<0.001^{\mathrm{c}}$ \\
\hline Negative symptoms & $9.73(4.79)^{\star}$ & $14.18(5.94)$ & - & $0.012^{c}$ \\
\hline General psychopathology & $23.68(9.02)^{* *}$ & $32.59(9.07)$ & - & $0.001^{\mathrm{c}}$ \\
\hline Total symptoms & $43.36(16.42)^{* * *}$ & $64.15(15.81)$ & - & $<0.001^{\mathrm{c}}$ \\
\hline \multicolumn{5}{|l|}{ BCSS } \\
\hline Negative self & $12.19(5.87)^{\dagger \dagger t}$ & $10.40(7.02)^{\dagger \dagger}$ & $3.81(8.26)$ & $<0.001$ \\
\hline Negative others & $9.79(6.32)^{t+t}$ & $9.80(7.00)^{t+\dagger}$ & $2.32(3.11)$ & $<0.001$ \\
\hline Positive self & $7.82(6.37)^{\dagger \dagger \dagger}$ & $8.68(5.49)^{\dagger \dagger \dagger}$ & $15.34(3.60)$ & $<0.001$ \\
\hline Positive others & $6.45(5.27)^{\dagger}$ & $7.28(5.61)$ & $10.52(5.19)$ & 0.015 \\
\hline \multicolumn{5}{|l|}{ Medication } \\
\hline Naive/free & $15 / 7$ & $8 / 8$ & - & - \\
\hline chlorpromazine equivalent (mg) & - & $348.89(218.67)(\mathrm{n}=9)$ & - & - \\
\hline
\end{tabular}

Table 1. Demographic and clinical characteristics of patients with APS, FES and HC. Data given as mean (SD). APS attenuated psychosis syndrome, BCSS brief core schema scale, FES first episode schizophrenia, HC healthy control, PANSS positive and negative syndrome scale. ${ }^{a}$ Significant $F$ statistic for the one way between group ANOVA. ${ }^{\mathrm{b}}$ Significant $\mathrm{F}$ statistic for the Chi-square test. ${ }^{\mathrm{c}}$ Significant $T$ statistic for the two sample $t$-test. ${ }^{\star} P<0.05,{ }^{\star \star} P<0.01,{ }^{* *} P<0.001$ compared to FES. ${ }^{\dagger} P<0.05,{ }^{\dagger \dagger} P<0.01,{ }^{\dagger \dagger} P<0.001$ compared to HC.

\begin{tabular}{|c|c|c|c|c|c|c|c|c|}
\hline Seed region & MNI coordinate & Cluster size & $t$ value & Effect size & $p$-FWE & $p$-FDR & $p$-unc & $\begin{array}{l}\text { Name (voxel size- } \\
\text { region) }\end{array}$ \\
\hline \multicolumn{9}{|l|}{ APS $>$ HC } \\
\hline $\begin{array}{l}\text { Right centromedial } \\
\text { amygdala }\end{array}$ & $-4638-2$ & 97 & 4.84 & 0.24 & 0.040 & 0.031 & 0.001 & $\begin{array}{l}60 \text { - left frontal pole } \\
\text { cortex }\end{array}$ \\
\hline \multirow{2}{*}{$\begin{array}{l}\text { Right laterobasal } \\
\text { amygdala }\end{array}$} & $12-26-26$ & 82 & 5.67 & 0.28 & 0.087 & 0.049 & 0.003 & 65-brain stem \\
\hline & $52-64-10$ & 78 & 5.75 & 0.23 & 0.104 & 0.049 & 0.003 & $\begin{array}{l}\text { 41-right inferior lat- } \\
\text { eral occipital cortex }\end{array}$ \\
\hline \multicolumn{9}{|l|}{ APS $<$ HC } \\
\hline $\begin{array}{l}\text { Right centromedial } \\
\text { amygdala }\end{array}$ & 0644 & 91 & 5.32 & 0.24 & 0.053 & 0.031 & 0.002 & $\begin{array}{l}\text { 66-right frontal pole } \\
\text { cortex }\end{array}$ \\
\hline \multicolumn{9}{|l|}{ FES $>$ APS } \\
\hline $\begin{array}{l}\text { Right superficial } \\
\text { amygdala }\end{array}$ & $-34-94-12$ & 98 & 5.53 & 0.23 & 0.044 & 0.048 & 0.001 & $\begin{array}{l}63 \text {-left occipital pole } \\
\text { cortex }\end{array}$ \\
\hline \multicolumn{9}{|l|}{ FES $>$ HC } \\
\hline $\begin{array}{l}\text { Left centromedial } \\
\text { amygdala }\end{array}$ & $-4-6-4$ & 97 & 5.98 & 0.19 & 0.040 & 0.034 & 0.001 & 16-left thalamus \\
\hline \multicolumn{9}{|l|}{ FES $<$ HC } \\
\hline $\begin{array}{l}\text { Right superficial } \\
\text { amygdala }\end{array}$ & $14-72-30$ & 139 & 4.93 & 0.21 & 0.008 & 0.009 & $<0.001$ & $\begin{array}{l}92 \text {-right cerebellum } \\
\text { crus1 }\end{array}$ \\
\hline
\end{tabular}

Table 2. Post-hoc analysis results of seed to voxel functional connectivity among APS $(n=22)$, FEP $(n=25)$, and $\mathrm{HC}(\mathrm{n}=31)$. Whole-brain thresholded at $p<0.05$, FDR corrected with a voxel extent of $>20$; Only coordinates of highest peak in each cluster are reported. 


\section{CMA}

(1) APS - HCS
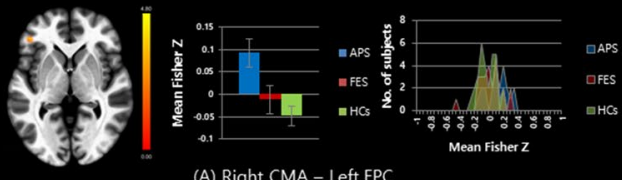

(A) Right CMA - Left FPC

Mean Fisher Z

(2) FES - HCs

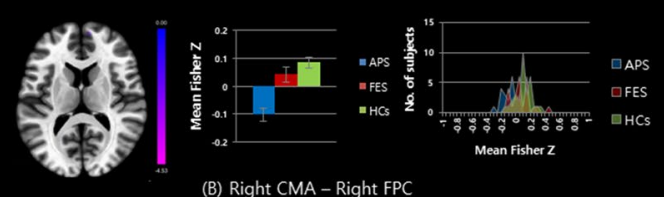

(B) Right CMA - Right FPC

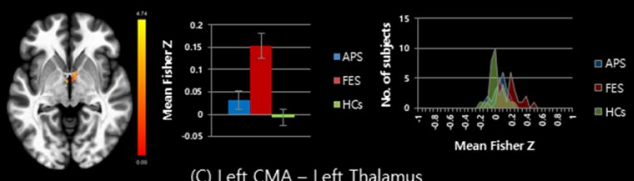

LBA

(1) APS - HCs
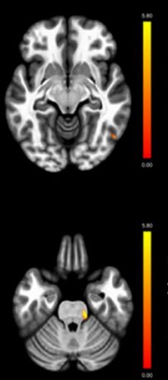

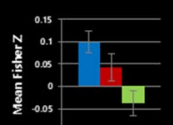

D) Right LBA - Brain stem

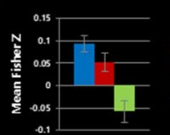

(E) Right LBA - Right $10 C$

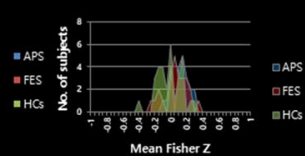

Mean Fisher Z

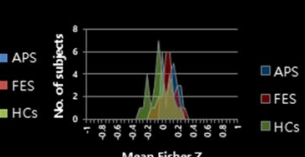

Mean Fisher Z
(1) APS - FES
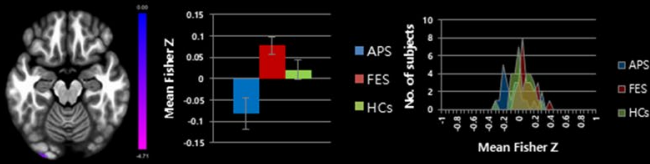

(F) Right SA - Left OPC

(2) FES - HCS

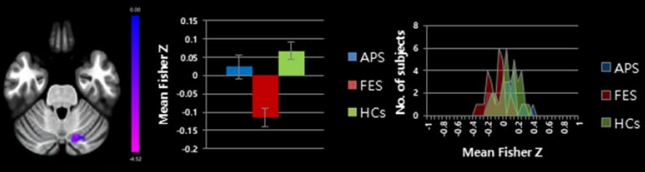

(G) Right SA - Right Cerebellum crus 1

Figure 1. Altered amygdala subregion-based functional connectivity among the attenuated psychosis syndrome, first episode schizophrenia and healthy control groups. Significant differences were revealed between the (A) Right centromedial amygdala (CMA) and Left frontal pole cortex (FPC); (B) Right CMA and Right FPC; (C) Left CMA and left thalamus; (D) Right laterobasal amygdala (LBA) and Brain stem; (E) Right LBA and Right inferior occipital cortex; (F) Right superficial amygdala (SA) and Left occipital pole cortex (OPC); (G) Right SA and Right cerebellum crus 1 . The functional connectivity $Z$ values of regions showing significant differences are presented in bar graph.

activation ${ }^{48}$ of the FPC. Considering the functions of the CMA and FPC, our finding suggests that individuals with APS are in an increased state of attention and vigilance to novel stimuli in the environment. This interpretation may be consistent with the hypothesis that psychosis is a state of aberrant assignment of salience to otherwise insignificant stimuli ${ }^{49}$.

Compared to HCs, individuals with APS were also found to have significant hyperconnectivity between the LBA and brain stem as well as the right inferior LOC. Increased connectivity of the LBA with the brain stem is supported by a previous study reporting that increased AMY connectivity with a brainstem was found in individuals at high risk for $\mathrm{SZ}^{19}$. The brainstem is home to a group of modulatory neurotransmitter pathways, such as those arising from the raphe nuclei (serotonergic), ventral tegmental area (dopaminergic), and locus coeruleus (noradrenergic), thereby controlling level-setting in sensory and arousal systems and emotional behaviors ${ }^{50}$. The LOC is involved in face perception ${ }^{51}$ or object recognition ${ }^{52}$. Therefore, our findings may be interpreted to indicate that individuals with APS who are exposed to negative facial stimuli are likely to exhibit physiological arousal. Unexpectedly, individuals with APS exhibited hypoconnectivity between the right CMA and right FPC compared to HCs. Several studies in which right FPC activity was measured via $\mathrm{fMRI}^{53}$ and $\mathrm{EEG}^{54}$ suggest that the right FPC plays a role in directed exploration. Hence, it may be that this decreased connectivity with right FPC could be associated with negative symptoms such as decreased exploration in individuals with APS. 
APS

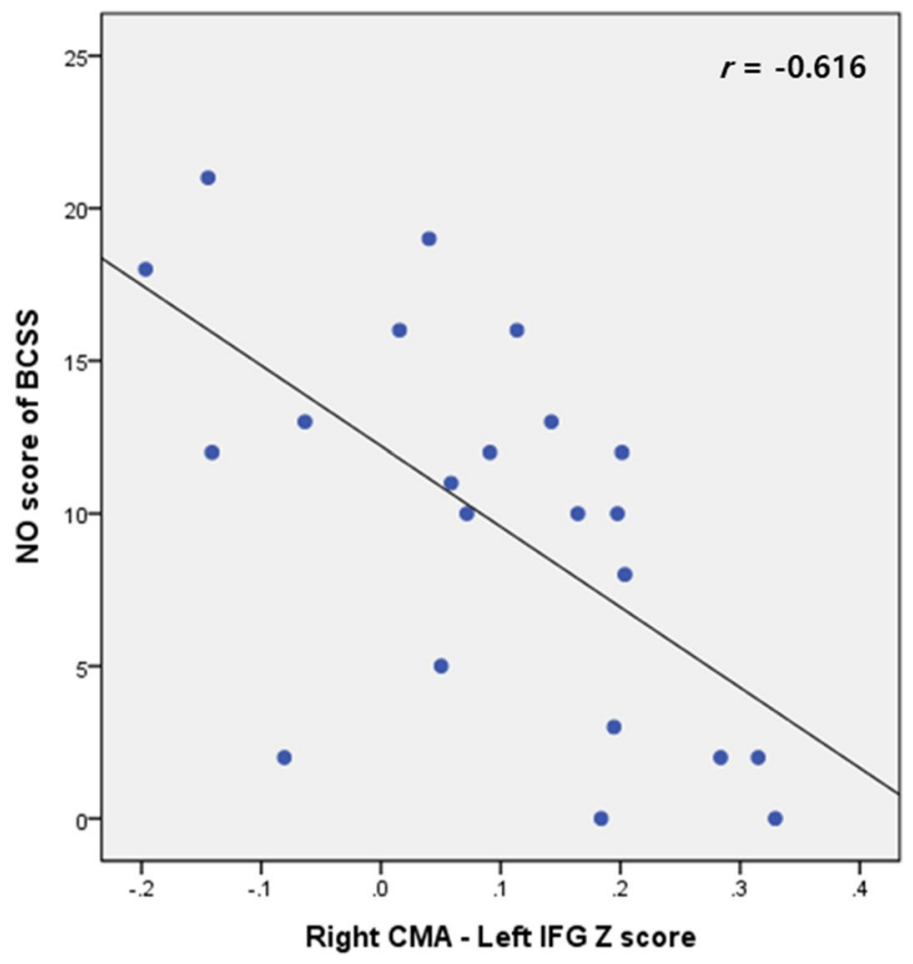

Figure 2. Associations between the right centromedial amygdala-left frontal pole cortex connectivity and negative-others scores of the Brief Core Schema Scale in the attenuated psychosis syndrome group.

In patients with FES, increased levels of connectivity were observed between the right SA and left OPC and between the left CMA and left thalamus compared to individuals with APS and HCs, respectively. The SA is implicated in olfactory and affective processes particularly related to facial emotions ${ }^{55}$. The occipital pole is the posterior-most part of the occipital lobe and is primarily responsible for visual processing. The traditional role of thalamus has been that of a passive relay station of information from sensory organs or subcortical structures to the cortex. However, its role has recently been extended to that of a critical hub region that could integrate diverse information being processed throughout the cerebral cortex as well as maintain the modular structure of cortical functional networks ${ }^{56}$. Increased connectivity between the AMY and thalamus in patients with FES is a relatively unique finding considering most of the previous studies on SZ reported decreased connectivity in amygdalo- ${ }^{34}$ or thalamo-cortical circuits ${ }^{57}$. Deficits in amygdala-orbitofrontal cortex coupling have been reported in both early course and chronic $\mathrm{SZ}^{19}$. Even though we did not observe altered amygdalo-cortical connectivity in FES group, our key finding is an aberrant connectivity between the AMY and sensory information processing regions, i.e., OPC and thalamus. This may suggest that individuals with FES are hypervigilant via sensory stimulation including facial perception. Moreover, it is in line with previous studies reporting strong connections between the AMY and auditory regions of the cortex and thalamus ${ }^{58}$ as well as disrupted pathways from limbic areas to thalamus in $\mathrm{SZ}^{59}$. Especially, it is interesting to note that strong connections between the AMY and auditory regions of the cortex and thalamus were shown in patients with SZ during the perception of aversive auditory stimuli mimicking the content of auditory verbal hallucinations ${ }^{58}$. The scores of P3 (hallucinatory behavior) in the PANSS were $1.82 \pm 1.01$ and $3.68 \pm 1.77$ for the APS and FES groups respectively. Therefore, it may be speculated that increased connectivity between the AMY and thalamus is associated with auditory verbal hallucinations in the FES group. It merits further investigation to see whether this aberrant connectivity between the AMY and thalamus is occurring in the APS group converted to psychosis.

Correlation analysis showed that the connectivity strength between the right CMA and left FPC was negatively related to the BCSS negative-others score in the APS group. This result seems counterintuitive considering our previous result from seed to voxel FC analysis, i.e. increased connectivity strength between the right CMA and left FPC in the APS group. A possible interpretation may be that although the strength of the connectivity between the CMA and FPC was stronger in individuals with APS than in HCs, greater connectivity is correlated with lower negative-others score within the APS group itself. Though clinical implication of inverse correlation within the APS group is ambiguous, it would be interesting to explore its relationship with conversion to psychosis. Alternatively, it may be that resting state brain activity does not reflect appropriately actual mental state, i.e., BCSS score and may be showing decompensated activity for negative schema. This study has several limitations. First, in the APS group, the sample size was small, and the sex ratio was male-biased. Second, because 
we used a cross-sectional design, there was no information about conversions to psychosis among individuals with APS. Therefore, the findings in APS should be interpreted cautiously. Third, some of the participants with FES were taking atypical antipsychotics, raising the possibility of a medication confound. Given the rapid and reversible effects of antipsychotic drugs on corticostriatal circuits ${ }^{60}$, future studies with drug-naive patients are required to eliminate medication effects.

Despite these caveats, this is the first trial to use three AMY subregions as seed ROIs to explore whole brain connectivity in individuals with APS. In conclusion, AMY-based voxel-wise analysis of FC with the rest of the brain demonstrated altered FC with the CMA and LBA in the APS group and the CMA and SA in the FES group compared to HCs. Especially for the CMA, increased connectivity with the FPC and thalamus was observed in the APS and FES groups, respectively. This suggests that the APS group may be hypervigilant to cognitive stimuli and FES group to sensory stimuli. Increased AMY connectivity with thalamus in the FES group may be associated with the development of auditory verbal hallucinations. Given the lack of specificity of many biological markers in psychiatric disorders, our findings suggest that FC analysis using a specific AMY subregion as a seed ROI could help uncover more specific neuroimaging biomarkers for psychosis.

\section{Methods}

Participants. Participants were help-seeking individuals with APS, patients with FES, and HCs. Diagnostic evaluation was performed by trained psychiatrists according to the Structured Clinical Interview for DSM-IV $(\text { SCID })^{61,62}$. For diagnosis and subtype classification of CHR states for psychosis, criteria of the DSM- $5^{63}$ and Comprehensive Assessment of At-Risk Mental States (CAARMS) ${ }^{64}$ were employed. We recruited only the subjects with APS because it was most extensively studied syndrome within the psychosis continuum. The duration of illness (DI) for APS was calculated since the first significant attenuated psychotic symptoms appeared. First episode was defined as having experienced only a single episode of psychosis after the onset (individuals relapsing with another psychotic episode were excluded) and a DI of $<2$ years. Patients or individuals with alcohol or drug use disorders within the past 6 months, intellectual disability (IQ $\leq 70)$, current or historical neurological disorders, a serious medical illness, pregnancy, and claustrophobia were excluded from the study. HCs were recruited via advertisements. The participants were interviewed using the screening module of the SCID-IV non-patient edition ${ }^{62}$ and were required to have no previous or current psychiatric disorders, neurological disorders, or significant medical conditions. Controls having a first-degree relative with a psychiatric disorder were also excluded. All participants were between 19 and 60 years of age and were assessed as right-handed using the Edinburgh Handedness Inventory ${ }^{65}$. They joined the study voluntarily and provided written informed consent. The study was approved by the Ethics Committee of Jeonbuk National University Hospital (approval number: CUH 2012-08-001). A statement confirming that all experiments were performed in accordance with relevant guidelines and regulations.

Clinical assessment. The severity of symptoms was evaluated within a week of fMRI scanning using the Scale of Prodromal Symptoms ${ }^{66,67}$ (SOPS) or PANSS ${ }^{40,41}$. These scales were administered by trained psychiatrists. In addition, the BCSS $^{42}$, a self-rating scale, was administered. The BCSS yields four subscale scores on negativeself, positive-self, negative-others, and positive-others schemas. A higher score indicates a greater endorsement of a schema. This scale was chosen on the basis of the close association between negative schemas and positive symptoms ${ }^{39}$.

Image acquisition and preprocessing. Resting-state functional and structural MRI data were obtained at the Jeonbuk National University Hospital on a $3 \mathrm{~T}$ Verio scanner (Siemens Magnetom Verio, Erlangen, Germany) using a 12-channel standard quadrature head coil. The three-dimensional T1-weighted image was acquired using magnetization-prepared rapid gradient echo (repetition time [TR]: $1900 \mathrm{~ms}$, echo time [TE]: $2.5 \mathrm{~ms}$; flip angle: $9^{\circ}$; field of view [FOV]: $250 \mathrm{~mm}$; image matrix: $256 \times 246 \mathrm{~mm}$; voxel size $=1.0 \times 1.0 \times 1.0 \mathrm{~mm}^{3}$; 176 slices). For each participant, we collected a 5-min resting-state scan consisting of 150 contiguous echo-planar imaging functional images (TR: $2000 \mathrm{~ms}$; TE: $30 \mathrm{~ms}$; flip angle: $90^{\circ}$; FOV: $220 \mathrm{~mm}$; image matrix: $64 \times 64 \mathrm{~mm}$; voxel size $=1.0 \times 1.0 \times 1.0 \mathrm{~mm}^{3} ; 26$ slices). During resting-state image acquisition, the participants were asked to relax with their eyes closed and not to sleep. MRI data processing was conducted using Statistical Parametric Mapping software package, version 12 (SPM12; www.fil.ion.ucl.ac.uk/spm; Wellcome Department of Cognitive Neurology, London, UK) implemented in MATLAB. The first three volumes were discarded to adjust for magnetization equilibrium effects. Functional images were slice-time corrected, realigned to correct for head motion artifacts, and co-registered with each participant's structural image. Then, the co-registered functional data were transformed into a standard anatomical space based on the parameters obtained by spatially normalizing each T1 image to the Montreal Neurological Institute (MNI) template. Normalized images were smoothed with an 8 -mm full-width at half-maximum isotropic Gaussian kernel. The criteria for excessive head motion were translation $>2 \mathrm{~mm}$ or rotation $>2^{\circ}$ in any direction and frame displacement (FD) values $>0.5 \mathrm{~mm}$. Participants for whom more than $10 \%$ of volumes showed excessive head motion were excluded from the analysis ${ }^{68}$. FD values were computed using the FSL toolbox (https://www.fmrib.ox.ac.uk/fsl/index.html). Head motion was measured in six dimensions and the component correction (CompCor) ${ }^{69}$ noise components were used as nuisance variables. The CompCor built into the CONN toolbox (V 14f., https://www.nitrc.org/projects/conn) was used to increase the accuracy of grey matter (GM) signals by removing physiological noises such as heart rate and breathing signals, followed by the removal of the main components from the white matter (WM) and cerebrospinal fluid (CSF) signals. Afterward, the linear trend was removed through the time course, and the band-pass filter $(0.008<\mathrm{f}<0.09 \mathrm{~Hz})$ was applied. 
Functional connectivity analysis. The SPM Anatomy toolbox ${ }^{70}$ (V 2.2c) was used to parcellate the AMY into three ROIs, i.e., CMA, LBA, and $\mathrm{SA}^{71}$. After applying predefined ROIs to the image data of each subject, we used MANGO (https://ric.uthscsa.edu/mango/mango.html) to check whether they matched well. For each ROI, the BOLD time series of the voxels within the ROI were averaged to generate the reference time series for the ROI. FC analysis was performed using correlation analysis between the seed amygdala ROI and other voxels of the entire brain in a voxel-wise manner using the CONN toolbox. Group comparison was performed using a one-way analysis of variance (ANOVA) with sex and education as covariates. Direct group comparisons were further evaluated using post-hoc $t$-tests. In both analyses, we applied the voxel-level height threshold of uncorrected $\mathrm{p}<0.001$ and the cluster-level extent threshold of $\mathrm{p}<0.05$, which was corrected for multiple comparisons using the false discovery rate (FDR). To control the probability of a type I error, we further performed 10,000 permutation tests implemented in the CONN toolbox.

Statistical analysis. Demographic and clinical data were compared among the three groups using ANOVA, $t$-test, or Chi-square test depending on the variables. For the correlation analyses, the Region of Interest Extraction Tool (https://software.incf.org/software/rex) in the CONN toolbox was used to extract Fisher's Z transformed signal intensity values for brain regions with significant group differences. Relationships between the PANSS, BCSS, or SOPS score and z-scores were explored using sex and education as covariates with SPSS 20.0. Multiple comparison corrections were performed using the Bonferroni correction.

Received: 7 July 2020; Accepted: 6 October 2020

Published online: 19 October 2020

\section{References}

1. Friston, K. J. \& Frith, C. D. Schizophrenia: a disconnection syndrome?. Clin. Neurosci. 3, 89-97 (1995).

2. Friston, K., Brown, H. R., Siemerkus, J. \& Stephan, K. E. The disconnection hypothesis (2016). Schizophr. Res. 176, 83-94 (2016).

3. Yu, Q. et al. Brain connectivity networks in schizophrenia underlying resting state functional magnetic resonance imaging. Curr. Top. Med. Chem. 12, 2415-2425 (2012).

4. Bluhm, R. L. et al. Spontaneous low-frequency fluctuations in the BOLD signal in schizophrenic patients: anomalies in the default network. Schizophr. Bull. 33, 1004-1012 (2007).

5. Bluhm, R. L. et al. Retrosplenial cortex connectivity in schizophrenia. Psychiat. Res. 174, 17-23 (2009).

6. Liang, M. et al. Widespread functional disconnectivity in schizophrenia with resting-state functional magnetic resonance imaging. NeuroReport 17, 209-213 (2006).

7. Zhou, Y. et al. Altered resting-state functional connectivity and anatomical connectivity of hippocampus in schizophrenia. Schizophr. Res. 100, 120-132 (2008).

8. Sorg, C. et al. Increased intrinsic brain activity in the striatum reflects symptom dimensions in schizophrenia. Schizophr. Bull. 39, 387-395 (2013).

9. Wolf, N. D. et al. Dysconnectivity of multiple resting-state networks in patients with schizophrenia who have persistent auditory verbal hallucinations. J. Psychiat. Neurosci. 36, 366-374 (2011).

10. Camchong, J., Mac Donald, A. W. 3rd., Bell, C., Mueller, B. A. \& Lim, K. O. Altered functional and anatomical connectivity in schizophrenia. Schizophr. Bull. 37, 640-650 (2011).

11. Rotarska-Jagiela, A. et al. Resting-state functional network correlates of psychotic symptoms in schizophrenia. Schizophr. Res. 117, 21-30 (2010).

12. Guo, W. et al. Abnormal default-mode network homogeneity in first-episode, drug-naive schizophrenia at rest. Prog. NeuroPsychopharmacol. Biol. Psychiatry 49, 16-20 (2014).

13. He, Z. et al. Aberrant intrinsic brain activity and cognitive deficit in first episode treatment-naive patients with schizophrenia. Psychol. Med. 43, 769-780 (2013).

14. Zhou, Y. et al. Functional dysconnectivity of the dorsolateral prefrontal cortex in first-episode schizophrenia using resting-state fMRI. Neurosci. Lett. 417, 297-302 (2007).

15. Guo, W. et al. Decreased resting-state interhemispheric coordination in first-episode, drug-naïve paranoid schizophrenia. Prog. Neuro-Psychopharmacol. Biol. Psychiatry 48, 14-19 (2014).

16. Lui, S. et al. Short-term effects of antipsychotic treatment on cerebral function in drug-naive first-episode schizophrenia revealed by "resting state" functional magnetic resonance imaging. Arch. Gen. Psychiatry 67, 783-792 (2010).

17. Mwansisya, T. E. et al. Task and resting-state fMRI studies in first-episode schizophrenia: a systematic review. Schizophr. Res. 189, 9-18 (2017).

18. Fusar-Poli, P. et al. Predicting psychosis meta-analysis of transition outcomes in individuals at high clinical risk. Arch. Gen. Psychiatry 69, 220-229 (2012).

19. Anticevic, A. et al. Amygdala connectivity differs among chronic, early course, and individuals at risk for developing schizophrenia. Schizophr. Bull. 40, 1105-1116 (2014).

20. Wang, H. et al. Patients with first-episode, drug-naive schizophrenia and subjects at ultra-high risk of psychosis shared increased cerebellar-default mode network connectivity at rest. Sci. Rep. 6, 26124. https://doi.org/10.1038/srep26124 (2016).

21. Shim, G. et al. Altered resting-state connectivity in subjects at ultra-high risk for psychosis: an fMRI study. Behav. BrainFunct. 6, 58 (2010).

22. Dandash, O. et al. Altered striatal functional connectivity in subjects with an at-risk mental state for psychosis. Schizophr. Bull. 40, 904-913 (2014).

23. Fornito, A. et al. Functional dysconnectivity of corticostriatal circuitry as a risk phenotype for psychosis. JAMA Psychiatry 70, 1143-1151 (2013).

24. Yoon, Y. B. et al. Altered fronto-temporal functional connectivity in individuals at ultra-high-risk of developing psychosis. PLoS ONE 10, e0135347 (2015).

25. Anticevic, A. et al. Association of thalamic dysconnectivity and conversion to psychosis in youth and young adults at elevated clinical risk. JAMA Psychiatry 72, 882-891 (2015).

26. LeDoux, J. E. The emotional brain, fear, and the amygdala. Cell Mol. Neurobiol. 23, 727-738 (2003).

27. Phelps, E. A. \& LeDoux, J. E. Contributions of the amygdala to emotion processing: from animal models to human behavior. Neuron 48, 175-187 (2005).

28. Davis, M. Neurobiology of fear responses: the role of the amygdala. J. Neuropsychiatry Clin. Neurosci. 9, 382-402 (1997).

29. Kapp, B. S., Supple, W. F. Jr. \& Whalen, P. J. Effects of electrical stimulation of the amygdaloid central nucleus on neocortical arousal in the rabbit. Behav. Neurosci. 108, 81-93 (1994). 
30. Heimer, L. \& Van Hoesen, G. W. The limbic lobe and its output channels: implications for emotional functions and adaptive behavior. Neurosci. Biobehav. Rev. 30, 126-147 (2006).

31. Price, J. L. Comparative aspects of amygdala connectivity. Ann. N. Y. Acad. Sci. 985, 50-58 (2003)

32. Gonzalez-Lima, F. \& Scheich, H. Classical conditioning of tone-signaled bradycardia modifies 2-deoxyglucose uptake patterns in cortex, thalamus, habenula, caudate putamen and hippocampal formation. Brain Res. 363, 239-256 (1986).

33. Hoptman, M. J. et al. Amygdalofrontal functional disconnectivity and aggression in schizophrenia. Schizophr. Bull. 36, 1020-1028 (2010).

34. Liu, H. et al. Differentiating patterns of amygdala-frontal functional connectivity in schizophrenia and bipolar disorder. Schizophr. Bull. 40, 469-477 (2014).

35. Wei, S. et al. Similarities and differences of functional connectivity in drug naïve, first-episode adolescent and young adult with major depressive disorder and schizophrenia. Sci. Rep. 7, 44316. https://doi.org/10.1038/srep44316 (2017).

36. Zhang, M. et al. Abnormal amygdala subregional-sensorimotor connectivity correlates with positive symptom in schizophrenia. Neuroimage Clin. 26, 102218. https://doi.org/10.1016/j.nicl.2020.102218 (2020).

37. Gee, D. G. et al. NAPLS Consortium. Altered age-related trajectories of amygdala-prefrontal circuitry in adolescents at clinical high risk for psychosis: a preliminary study. Schizophr. Res. 134, 1-9 (2012).

38. Disner, S. G., Beevers, C. G., Haigh, E. A. \& Beck, A. T. Neural mechanisms of the cognitive model of depression. Nat. Rev. Neurosci. 12, 467-477 (2011).

39. Beck, A. T., Himelstein, R. \& Grant, P. M. In and out of schizophrenia: activation and deactivation of the negative and positive schemas. Schizophr. Res. 203, 55-61 (2019).

40. Kay, S. R., Fiszbein, A. \& Opler, L. A. The positive and negative syndrome scale (PANSS) for schizophrenia. Schizophr. Bull. 13, 261-276 (1987).

41. Yi, J. S. et al. Reliability and validity of the Korean version of the positive and negative syndrome scale. J. Korean Neuropsychiatr. Assoc. 40, 1090-1105 (2001).

42. Fowler, D. et al. The brief core schema scales (BCSS): psychometric properties and associations with paranoia and grandiosity in non-clinical and psychosis samples. Psychol. Med. 36, 749-759 (2006).

43. Dringenberg, H. C. \& Vanderwolf, C. H. Cholinergic activation of the electrocorticogram: an amygdaloid activating system. Exp. Brain. Res. 108, 285-296 (1996).

44. Goldstein, L. E., Rasmusson, A. M., Bunney, B. S. \& Roth, R. H. Role of the amygdala in the coordination of behavioral, neuroendocrine, and prefrontal cortical monoamine responses to psychological stress in the rat. J. Neurosci. 16, 4787-4798 (1996).

45. Schacter, D. L., Addis, D. R. \& Buckner, R. L. Remembering the past to image in the future: the prospective brain. Nat. Rev. Neurosci. 8, 657-661 (2007).

46. Mansouri, F. A., Buckley, M. J., Mahboubi, M. \& Tanaka, K. Behavioral consequences of selective damage to frontal pole and posterior cingulate cortices. PNAS 112, 3940-3949 (2015).

47. Rosso, I. M. et al. Regional prefrontal cortex gray matter volumes in youth at familial risk for schizophrenia from the Harvard Adolescent High Risk Study. Schizophr. Res. 123, 15-21 (2010).

48. den Braber, A. et al. Brain activation during cognitive planning in twins discordant or concordant for obsessive-compulsive symptoms. Brain 133, 3123-3140 (2010).

49. Kapur, S. Psychosis as a state of aberrant salience: a framework linking biology, phenomenology, and pharmacology in schizophrenia. Am. J. Psychiatry 160, 13-23 (2003).

50. Venkatraman, A., Ddlow, B. L. \& Yang, M. H. I. The brainstem in emotion: a review. Front. Neuroanat. 11, 15 (2017).

51. Nagy, K., Mark, G. \& Gyula, K. The lateral occipital cortex in the face perception network: an effective connectivity study. Front. Psychol. 3, 141 (2012).

52. Grill-Spector, K., Kourtzi, Z. \& Kanwisher, N. The lateral occipital complex and its role in object recognition. Vision Res. 41, 1409-1422 (2001).

53. Badre, D., Doll, B. B., Long, N. M. \& Frank, M. J. Rostrolateral prefrontal cortex and individual differences in uncertainty-driven exploration. Neuron 73, 595-607 (2012).

54. Cavanagh, J. F., Bismark, A. J., Frank, M. J. \& Allen, J. J. Larger error signals in major depression are associated with better avoidance learning. Front. Psychol. 2, 331 (2011).

55. Goossens, L. et al. Selective processing of social stimuli in the superficial amygdala. Hum. Brain Mapp. 30, 3332-3338 (2009).

56. Hwang, K., Bertolero, M. A., Liu, W. B. \& D’Esposito, M. The human thalamus is an integrative hub for functional brain networks. J. Neurosci. 37, 5594-5607 (2017).

57. Steullet, P. Thalamus related anomalies as candidate mechanism based biomarkers for psychosis. Schizophr. Res. https://doi. org/10.1016/j.schres.2019.05.027 (2019).

58. Horga, G. et al. Brain metabolism during hallucination-like auditory stimulation in schizophrenia. PLoS ONE 9, e84987 (2014).

59. Hua, M. et al. Disrupted pathways from limbic areas to thalamus in schizophrenia highlighted by whole-brain resting-state effective connectivity analysis. Prog. Neuropsychopharmacol. Biol. Psychiatry 99, 109837 (2020).

60. Tost, H. et al. Acute $\mathrm{D}_{2}$ receptor blockade induces rapid, reversible remodeling in human cortical-striatal circuits. Nat. Neurosci. 13, 920-922 (2010).

61. First, M.B., Spitzer, R.L., Gibbon, M., \& Williams, J.B.W. Structured clinical interview for DSM-IV axis I disorders (Patient ed. (SCID-P)) (Version 2). (New York, 1996).

62. Han OS, Hong JP. Structured clinical interview for DSM-IV axis I disorders, Research version. (Seoul, 2000).

63. American Psychiatric Association. Diagnostic and Statistical Manual of Mental Disorders: DSM-5.(Arlington, 2013)

64. Yung, A. R. et al. Mapping the onset of psychosis: the comprehensive assessment of at-risk mental states. Aust. N. Z. J. Psychiatry 39, 964-971 (2005).

65. Oldfield, R. C. The assessment and anlysis of handedness: the Edingburgh inventory. Neuropsychologia 9, 97-113 (1971).

66. Miller, T. J. et al. Prodromal assessment with the structured interview for prodromal syndromes and the scale of prodromal symptoms: predictive validity, interrater reliability, and training to reliability. Schizophr. Bull. 29, 703-715 (2003).

67. Jung, M. H. et al. The reliability and validity of the korean version of the structured interview for prodromal syndrome. Psychiatry Investig. 7, 257-263 (2010).

68. Heleven, E. \& Overwalle, F. V. The person within: memory codes for persons and traits using fMRI repetition suppression. Soc. Cogn. Affect. Neurosci. 11, 159-171 (2016).

69. Behzadi, Y., Restom, K., Liau, J. \& Liu, T. T. A component based noise correction method (CompCor) for BOLD and perfusion based fMRI. Neuroimage. 37, 90-101 (2007).

70. Eickhoff, S. B. et al. A new SPM toolbox for combining probabilistic cytoarchitectonic maps and functional imaging data. Neuroimage. 25, 1325-1335 (2005).

71. Amunts, K. et al. Cytoarchitectonic mapping of the human amygdala, hippocampal region and entorhinal cortex: intersubject variability and probability maps. Anat. Embryol. 210, 343-352 (2005).

\section{Acknowledgements}

The corresponding author would like to thank all participants in the study and father for guidance and support. 


\section{Author Contributions}

All authors provided critical input and revisions.

\section{Funding}

This study was supported by a grant of the Korean Mental Health Technology R\&D Project, Ministry of Health \& Welfare, Republic of Korea (HL19C0015) and a grant of the Korea Health Technology R\&D Project through the Korea Health Industry Development Institute (KHIDI), funded by the Ministry of Health \& Welfare, Republic of Korea (HI18C2383).

\section{Competing interests}

The authors declare no competing interests.

\section{Additional information}

Correspondence and requests for materials should be addressed to Y.-C.C.

Reprints and permissions information is available at www.nature.com/reprints.

Publisher's note Springer Nature remains neutral with regard to jurisdictional claims in published maps and institutional affiliations.

(c) (i) Open Access This article is licensed under a Creative Commons Attribution 4.0 International License, which permits use, sharing, adaptation, distribution and reproduction in any medium or format, as long as you give appropriate credit to the original author(s) and the source, provide a link to the Creative Commons licence, and indicate if changes were made. The images or other third party material in this article are included in the article's Creative Commons licence, unless indicated otherwise in a credit line to the material. If material is not included in the article's Creative Commons licence and your intended use is not permitted by statutory regulation or exceeds the permitted use, you will need to obtain permission directly from the copyright holder. To view a copy of this licence, visit http://creativecommons.org/licenses/by/4.0/.

(c) The Author(s) 2020 